\title{
Experimental context modulates warning signal effects
}

\author{
W. Machado-Pinheiro ${ }^{1}$, \\ A.J.P. Faria Jr. ${ }^{1}$, \\ L.G. Gawryszewski² \\ and L.E. Ribeiro-do-Valle ${ }^{3}$
}

\author{
'Laboratório de Neurofisiologia do Comportamento, \\ Departamento de Fisiologia e Farmacologia, Instituto Biomédico, and \\ ${ }^{2}$ Laboratório de Neurobiologia da Atenção, Departamento de Neurobiologia, \\ Instituto de Biologia, Universidade Federal Fluminense, Niterói, RJ, Brasil \\ ${ }^{3}$ Departamento de Fisiologia e Biofísica, Instituto de Ciências Biomédicas, \\ Universidade de São Paulo, São Paulo, SP, Brasil
}

\section{Correspondence \\ W. Machado-Pinheiro Laboratório de Neurofisiologia \\ do Comportamento \\ Departamento de Fisiologia \\ e Farmacologia \\ Instituto Biomédico, UFF \\ Rua Hernani Mello, 101 \\ 24210-130 Niterói, RJ \\ Brasil \\ Fax: +55-21-2618-3389 \\ E-mail: walter@vm.uff.br \\ Presented at the XVII Annual Meeting of the Federação de Sociedades de Biologia Experimental, Salvador, BA, Brazil, August 28-31, 2002. \\ Research supported by PRONEX/MCT, CAPES, CNPq and PROPP-UFF. A.J.P. Faria Jr. is the recipient of a CAPES fellowship.}

Publication supported by FAPESP.

Received September 12, 2003

Accepted April 26, 2004

\begin{abstract}
Previous studies have shown that saccadic eye responses but not manual responses were sensitive to the kind of warning signal used, with visual onsets producing longer saccadic latencies compared to visual offsets. The aim of the present study was to determine the effects of distinct warning signals on manual latencies and to test the premise that the onset interference, in fact, does not occur for manual responses. A second objective was to determine if the magnitude of the warning effects could be modulated by contextual procedures. Three experimental conditions based on the kind of warning signal used (visual onset, visual offset and auditory warning) were run in two different contexts (blocked and non-blocked). Eighteen participants were asked to respond to the imperative stimulus that would occur some milliseconds $(0,250$, 500 or $750 \mathrm{~ms}$ ) after the warning signal. The experiment consisted in three experimental sessions of 240 trials, where all the variables were counterbalanced. The data showed that visual onsets produced longer manual latencies than visual offsets in the nonblocked context (275 vs $261 \mathrm{~ms}$; P < 0.001). This interference was obtained, however, only for short intervals between the warning and the stimulus, and was abolished when the blocked context was used (256 vs $255 \mathrm{~ms} ; \mathrm{P}=0.789$ ). These results are discussed in terms of bottom-up and top-down interactions, mainly those related to the role of attentional processing in canceling out competitive interactions and suppressive influences of a distractor on the relevant stimulus.
\end{abstract}

Key words

- Reaction times

- Attention

- Distractor

- Preparation

- Expectancy

........................
There has been general agreement that reaction times are reduced when a warning signal (WS) precedes the onset of a subsequent stimulus - the imperative stimulus (IS) - to which a response must be given. Nevertheless, some studies have shown that the facilitation induced by the WS can be modu- lated by the kind of WS employed; for example, visual onset, visual offset or auditory warning. In two classical papers, Ross and Ross $(1,2)$ showed that visual onset and visual offset used as WS produced different effects on saccade latency: saccadic reaction times obtained after visual offset were shorter 
than those obtained after visual onset. Moreover, they showed that the difference between offset and onset was only observed at the short intervals of 0 and $100 \mathrm{~ms}$. For longer intervals, no significant differences occurred (1). However, when manual responses were required (to move a vertical lever to the right or to the left), no significant difference was found between visual onset and offset as WS, leading these investigators to propose that differential effects were specific for eye movements: the onset of a visual WS would have an interfering effect on the programming or execution of a saccade, but not of a manual response. In fact, many other investigators, using quite different experimental procedures, have confirmed this interference of visual onsets with saccadic responses $(3,4)$.

However, our group has found some evidence of distinct effects between visual onset and offset also using manual reaction time (MRT) $(5,6)$. In these reports a noninformative visual cue (S1) preceded the IS, which could be presented $4^{\circ}$ to the left or to the right of a fixation point (FP), ipsilaterally or contralaterally to $\mathrm{S} 1$. The non-informative $\mathrm{S} 1$ could be the onset or the offset of a visual stimulus $6^{\circ}$ to the left or to right of the FP. Our results showed that the onset and the offset of the cues had distinct effects on manual latencies: MRTs at short intervals were faster for contralateral offset cues than for contralateral onset cues. We interpreted this as evidence that the non-informative visual onset is able to attract automatically the participant's attention and so to interfere with manual responses to contralateral stimuli. Thus, in contrast to the results obtained by Ross and Ross (1), we showed that manual responses can also be sensitive to the use of visual onset or offset. Despite the differences in the experimental procedures, the results obtained by Ross and Ross and those obtained by our group point to a possible interfering mechanism common to both saccadic and manual responses mediated by attentional processes.

The aim of the present study was to determine the effects of distinct WS on manual responses to visual stimuli. We tested the premise proposed by Ross and Ross $(1,2)$ that onset and offset of a visual warning has a similar effect on manual responses. As a second goal, we determined whether the magnitude of the warning effects is modulated by contextual procedures. In this case, a possible contextual effect generated by the use of a blocked or non-blocked (random) arrangement of WS during the experiment was tested. Moreover, since Ross and Ross (1) showed that differences between onset and offset, for saccadic latencies, were obtained only at short intervals, we tested whether this effect is, in fact, modulated by the interval between WS and IS.

Eighteen undergraduate students (18 to 27 years old) volunteered to participate in the experiment. Participants were right-handed according to the Edinburgh Inventory (7), had normal or corrected vision and were naive with respect to the goals of the experiment. The tasks occurred in a sound-attenuated room under dim ambient light. Participants sat in front of a 14-inch video monitor $\left(0.2 \mathrm{~cd} / \mathrm{m}^{2}\right)$, with their head positioned on a chin-and-forehead rest with their eyes positioned $57 \mathrm{~cm}$ from the screen. The timing of the presentation of stimuli and the recording of key pressings were performed by a PC486 microcomputer running customized software written in MEL2 language (Psychology Software Tools, Pittsburgh, PA, USA). The Hospital Ethics Committee approved the procedures adopted, and written informed consent was obtained from each volunteer prior to the study.

Participants were initially submitted to a short training session in which the importance of maintaining fixation was stressed. With a suitably positioned mirror, adherence to this instruction was checked. Shifts in gaze prior to the manual response turned out to be very rare. The three experimental con- 
ditions used were defined by the kind of WS adopted. Each trial began with the presentation of a FP at the center of the screen. Seven hundred milliseconds later, one of three possible WS occurred: i) condition BEEP - a 2000-Hz, 67-dB, 50-ms tone ("beep") was emitted by a loudspeaker placed directly below the screen; ii) condition $\mathrm{ON}$ - a circle (radius of $0.5^{\circ}$ and brightness of $7.5 \mathrm{~cd} / \mathrm{m}^{2}$ ) centered on the FP was turned on; iii) condition OFF - a circle, initially present, centered on the FP, was turned off. For each condition, after a variable interval following the WS $(0,250,500$ or $750 \mathrm{~ms})$, a white disk $\left(7.5 \mathrm{~cd} / \mathrm{m}^{2}\right.$ - the IS) appeared on the screen $8^{\circ}$ to the right of the FP. WS and IS remained on until the execution of the motor response. The intervals were randomized and occurred with the same probability throughout the experiment. Moreover, the conditions were arranged in two possible contexts: blocked and non-blocked. In the blocked context each condition was run in a different block of trials. In the non-blocked context the three conditions were randomized throughout the experiment.

Participants were told to use the WS as an indicator that IS was imminent, and to press a key with their right index finger as soon as they detected IS. At the end of each trial the MRT immediately appeared on the screen and remained on for $1000 \mathrm{~ms}$ until the next trial. Trials in which the MRTs were shorter than $100 \mathrm{~ms}$ or longer than $700 \mathrm{~ms}$ were considered erroneous and volunteers were informed with the messages "anticipation" or "slow response", respectively. Error trials were repeated at the end of the session. Participants performed three sessions on separate days (approximately $30 \mathrm{~min}$ per session). The session consisted of three blocks of 80 trials each, with 1 or 2 min of rest between blocks. Data collected on the first day were considered to be part of training and were not analyzed. In this first session, participants were presented to the three conditions and to both blocked and non-blocked contexts. In the second and third sessions "context" and "condition" were counterbalanced across subjects, in such a way that all of them were run equally throughout the experiment.

MRT was the dependent variable. The medians of the data obtained in the second and third sessions were submitted to an analysis of variance (ANOVA) in which interval (0, 250, 500 and $750 \mathrm{~ms})$, condition (BEEP, OFF and ON) and context (blocked and non-blocked) were used as within-participant factors. Planned comparisons were employed in order to test for relevant differences among levels, mainly those related to the comparisons between $\mathrm{ON}$ and OFF conditions.

The errors corresponded to $3.3 \%$ and most of them were anticipation $(81 \%)$. They tended to be equally distributed among subjects and across days, but were more numerous for the longer intervals than for the shorter ones: 72 errors occurred at the intervals of 0 and $250 \mathrm{~ms}$, while 212 occurred at the intervals of 500 and $750 \mathrm{~ms}$.

ANOVA showed a main effect of context $\left(\mathrm{F}_{1,17}=5.52, \mathrm{P}=0.031\right)$, condition $\left(\mathrm{F}_{2,34}=\right.$ $4.48, \mathrm{P}=0.018)$ and interval $\left(\mathrm{F}_{3,51}=136.88\right.$, $\mathrm{P}<0.001)$. MRTs obtained for the blocked context were smaller than those for the nonblocked context (254 vs $265 \mathrm{~ms}$ ); also, MRTs for the ON condition (265 ms) were longer than those obtained for the OFF (258 $\mathrm{ms}$ ) and BEEP (256 ms) conditions. The interaction between condition and interval was significant $\left(\mathrm{F}_{6,102}=4.92, \mathrm{P}<0.001\right)$ and that between context and condition was also marginally significant $\left(\mathrm{F}_{2,34}=2.89, \mathrm{P}=\right.$ 0.069 ). The other interactions were not significant. The interaction between condition and interval occurred due to differences among conditions at short intervals: at 0 - and 250-ms interval, MRTs for the ON condition were longer than the others. However, for the intervals of 500 and $750 \mathrm{~ms}$ no differences occurred between conditions.

Figure 1 shows that in the non-blocked 
Figure 1. Mean latency of manual reaction times (ms) as a function of condition (BEEP, OFF and $\mathrm{ON}$ ) and context (blocked and non-blocked). The error bars indicate \pm SEM. Planned comparisons showed that in the non-blocked context reaction times for the ON condition were longer than those obtained for the OFF condition ( ${ }^{*} \mathrm{P}$ $<0.05)$.

Figure 2. Mean latency of manual reaction times (ms) as a function of interval (ms) and condition (BEEP, OFF and ON) for the two contexts: $A$, blocked context; $B$, non-blocked context. The error bars indicate \pm SEM. Planned comparisons showed that in the non-blocked context reaction times for the ON condition were longer than those obtained for the OFF condition at the intervals of 0 and $250 \mathrm{~ms}\left({ }^{*} \mathrm{P}<0.05\right)$. context, MRTs for the ON condition (275 $\mathrm{ms}$ ) were longer than those for the OFF (261 $\mathrm{ms}$ ) and BEEP (260 ms) conditions. However, in the blocked context MRTs for the three conditions did not differ (256, 255 and $252 \mathrm{~ms}$, respectively). Planned comparisons confirmed that the difference between the $\mathrm{ON}$ and OFF conditions observed in Figure 1 was significant in the non-blocked context $\left(\mathrm{F}_{1,17}=17.13, \mathrm{P}<0.001\right)$, but not in the blocked context $\left(\mathrm{F}_{1,17}=0.07, \mathrm{P}=0.789\right)$. Figure 2 shows the variation of MRTs as a function of condition, context and interval. These data suggest that the modulation of MRTs observed in the ON condition was mainly due to an increase in motor latencies at the short intervals ( 0 and $250 \mathrm{~ms}$ ) of the non-blocked context. The planned comparisons between $\mathrm{ON}$ and OFF conditions performed for all tested intervals of both contexts confirmed this prediction. Significant differences were only obtained in the non-
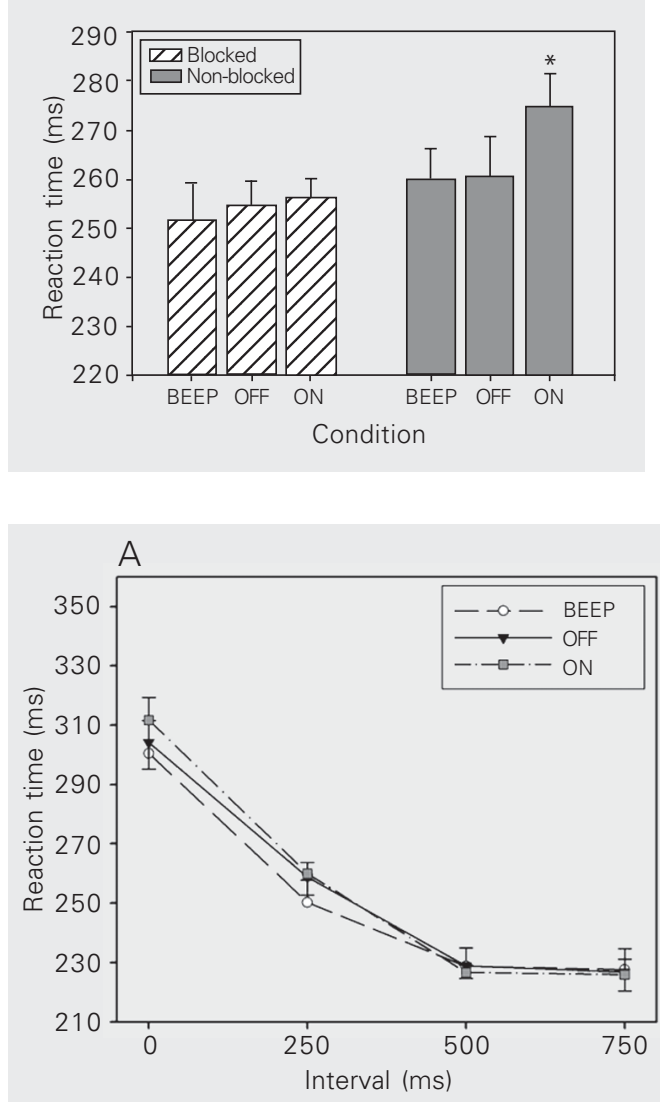

blocked context, at the intervals of $0 \mathrm{~ms}$ ( 337 vs $306 \mathrm{~ms}$, respectively for $\mathrm{ON}$ and OFF conditions; $\left.\mathrm{F}_{1,17}=23.49, \mathrm{P}<0.001\right)$ and 250 $\mathrm{ms}\left(282\right.$ vs $267 \mathrm{~ms}$, respectively; $\mathrm{F}_{1,17}=$ $5.08, \mathrm{P}=0.037)$. All other comparisons were nonsignificant: non-blocked/500 ms (241 vs $237 \mathrm{~ms} ; \mathrm{P}=0.228$ ); non-blocked $/ 750 \mathrm{~ms}$ (238 vs 232 ms; $\mathrm{P}=0.132$ ); blocked $/ 0 \mathrm{~ms}$ (312 vs $304 \mathrm{~ms} ; \mathrm{P}=0.338$ ); blocked $/ 250 \mathrm{~ms}$ (260 vs $259 \mathrm{~ms} ; \mathrm{P}=0.861$ ); blocked $/ 500 \mathrm{~ms}$ (227 vs $229 \mathrm{~ms} ; \mathrm{P}=0.700$ ), and blocked/750 $\mathrm{ms}$ (226 vs $227 \mathrm{~ms} ; \mathrm{P}=0.882)$. Therefore, the present data indicate that the above mentioned interaction between condition and interval was due to differences found within the non-blocked context, at short intervals.

The present results confirm the reports that distinct WS produce different effects on MRTs and also that these effects can be modulated by changes in the experimental context (blocked vs non-blocked). No difference among conditions was apparent in the blocked context: when participants perceived that the WS did not change in a block of trials, they were able to equally use any kind of WS in order to improve their performance. Conversely, in the non-blocked array, differences among conditions occurred: MRTs for the ON condition increased in relation to the others. Thus, when participants cannot foresee the kind of WS, a visual onset around the FP produces a disturbing effect and so reduces the participants' per-

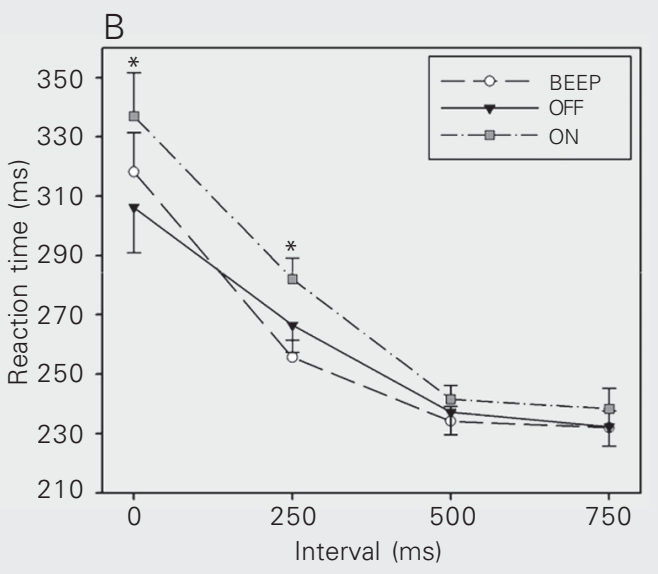


formance. Figure 2 shows that the interference obtained for the ON condition was observed only at short intervals. At the intervals of 500 and $750 \mathrm{~ms}$, MRTs for the three conditions did not differ. This suggests that the $\mathrm{ON}$ interference is a transient phenomenon, which vanishes as the interval increases.

These results confirm that distinct WS can produce different effects also on manual responses. Again, our data do not support the report by Ross and Ross $(1,2)$ that only the oculomotor system is sensitive to the interfering effects produced by visual onsets. In fact, data obtained by our group in another study with saccade latency revealed the same pattern of interference (8). In that study, a very similar paradigm was used. The only difference was the inclusion of a fourth condition, a "no-warning" one, in which the WS was absent. Saccadic responses increased for the $\mathrm{ON}$ condition in relation to the OFF condition. This interference was significant at $0 \mathrm{~ms}$ ( 271 vs $252 \mathrm{~ms}$, respectively, for the ON and OFF conditions) and $250 \mathrm{~ms}$ (253 vs $237 \mathrm{~ms}$, respectively). Saccadic latency obtained at $500 \mathrm{~ms}$ (221 vs $216 \mathrm{~ms}$ ) and $750 \mathrm{~ms}$ (217 vs $205 \mathrm{~ms}$ ) did not differ significantly. Therefore, our saccadic data reproduced exactly the same pattern as obtained for manual responses: ON interference was present at short intervals. A possible reason for the difference between our data and those reported by Ross and Ross $(1,2)$ is related to the use of the blocked condition in their procedures. As our data showed, the use of a blocked array can mitigate or even eliminate the occurrence of differences between onset and offset visual warnings.

On the basis of our data, we propose that the interference observed when the visual onset warning was used represents a general effect related to attentional mechanisms, which is able to influence both motor systems. In our view, the visual onset splits the participants' processing resources between the two stimuli: the foveal ON (WS) and the
IS. In other words, attention is automatically attracted to the foveal onset, reducing the central processing of IS. In fact, there is considerable evidence that an abrupt onset does capture attention in an automatic or passive fashion (e.g., 9,10), and also that visual onsets have priority over visual offset in the spatial orienting of attention $(11,12)$. The interference with behavioral performance occurred only at short intervals, when the time lags between WS and IS were not sufficient to allow an "adequate" processing of both stimuli. We believe that, as the interval increased, the probability of IS occurrence also increased, and so, top-down influences - here called expectancy - would overcome the transient bottom-up interference due to the foveal onset. Thus, at long intervals, preparation for IS occurrence (expectancy) would be maximal and its effects would overcome the $\mathrm{ON}$ interference. Interestingly, the use of the blocked context generated a similar phenomenon. In the nonblocked context, differences between $\mathrm{ON}$ and OFF were found at $0-$ and $250-\mathrm{ms}$ intervals (31 and $15 \mathrm{~ms}$, respectively). This difference was abolished in the blocked context (8 and $1 \mathrm{~ms}$ ). When the blocked context was used, and the WS was constant, the bottom-up interference induced by the visual onset was reduced or even abolished. Our data suggest that two different top-down influences are able to interact and sometimes overcome the transient ON effect, namely, i) time probability of IS occurrence - expectancy, and ii) certainty about the kind of WS to be used - the context.

Electrophysiological studies conducted by Rizzolatti in cats (see 9, for details) and by Judge and co-workers in monkeys (e.g., 13) showed that cellular responses to visual stimuli in both primary visual cortex and superior colliculus were strongly decreased when another visual stimulus was presented either inside or outside the receptive field of the cell. The inhibition occurred only for simultaneous or temporally close stimuli. We believe 
that our results represent the behavioral expression of such suppressive mechanisms. More recently, electrophysiological studies on monkeys (14) and neuroimaging studies $(15,16)$ have confirmed such influence in higher visual centers. Briefly, those studies showed that these suppressive interactions can be canceled out, at least partially, by the voluntary orienting of attention - a top-down bias. Moreover, Kastner et al. (16) described an expectancy-related activity in many cortical areas, which appeared when participants were directing their attention at a peripheral target location, expecting stimulus occurrence there. Expectancy-related activity was significantly stronger in frontal (frontal eye field and supplementary eye field) and parietal (superior parietal lobule) areas than in those directly involved in visual processing (V1, V2 and V4). They proposed that it reflects a top-down bias from frontal and parietal areas in order to allocate attentional resources and so cancels out suppressive interactions between simultaneous stimuli. Corchs and Deco (17) confirmed these findings in an elegant computational neuroscience study. They showed that not only the spatial biasing (dorsal pathway) but also the objectfeature biasing (ventral pathway) can be used to introduce some top-down bias (attentional) in order to cancel out the suppressive inhibition coming from simultaneous visual presentation.

In our experiment, the spatial allocation of attention was not a critical point, since the position of IS was constant. Our experimental procedures induce participants to use the moment of IS occurrence - temporal predictability - as a major source of influence on motor performance. This kind of top-down influence was already proposed by some investigators $(18,19)$ and, in our view, it is linked to a motor preparation that follows the WS, being strongly dependent on precise timing of IS - temporal expectancy. In reaction time experiments, motor-preparation activity occurs during the foreperiod and contributes to shortening the motor latencies. In fact, the temporal allocation of attention has recently received the proper recognition (e.g., 19,20) as a major factor modulating reaction times.

The present paper showed that the temporal allocation of attention (expectancy) and the repetitive presentation of a visual onset as warning (the blocked context) were capable of partially or completely abolishing the onset interference. Thus, our data complement and extend those mentioned above, showing two other top-down mechanisms that can be a source of attentional bias able to cancel out competitive interactions and suppressive influences of a distractor on the relevant stimulus. The results presented here, together with others (Machado-Pinheiro W, Faria Jr. AJP, Gawryszewski LG and Ribeirodo-Valle LE, unpublished data; Faria Jr. AJP and Machado-Pinheiro W, unpublished data) emphasize the importance of contextual manipulations and the interactions between bottom-up and top-down influences on MRTs.

\section{Acknowledgments}

We would like to thank Dr. Ronald Ranvaud and Dr. Marcus Vinícius C. Baldo, from Instituto de Ciências Biomédicas, Universidade de São Paulo, for their valuable comments on an early version of the manuscript.

\section{References}

1. Ross EL \& Ross SM (1980). Saccade latency and warning signals: stimulus onset, offset and change as warning events. Perception and Psychophysics, 27: 251-257.
2. Ross SM \& Ross LE (1981). Saccade latency and warning signals: effects of auditory and visual stimulus onset and offset. Perception and Psychophysics, 29: 429-437. 
3. Braun D \& Breitmeyer BG (1990). Effects of reappearance of fixated and attended stimuli upon saccadic reaction time. Experimental Brain Research, 81: 318-324.

4. Walker R, Deubel H, Schneider WX \& Findlay JM (1997). Effect of remote distractor on saccade programming: evidence for an extended fixation zone. Journal of Neurophysiology, 78: 1108-1119.

5. Gawryszewski LG, Thomaz TG, Machado-Pinheiro W \& Sant'Anna AN (1994). Onset and offset of a visual cue have different effects on manual reaction time to a visual target. Brazilian Journal of Medical and Biological Research, 27: 67-73.

6. Carreiro LRR, Gawryszewski LG, Magalhães FV \& Tradardi V (1994). Onset and offset inhibitions: effects of increase and decrease of cue luminance on manual reaction time to a visual target. Brazilian Journal of Medical and Biological Research, 27: 2645-2651.

7. Oldfield RC (1971). The assessment and analysis of handedness: The Edinburgh Inventory. Neuropsychologia, 9: 97-113.

8. Machado-Pinheiro W, Gawryszewski LG \& Ribeiro-do-Valle LE (1998). Efeitos de diferentes alertantes sobre os sistemas oculomotor e manual. XIII Annual Meeting of the Federação de Sociedades de Biologia Experimental, Caxambu, MG, Brazil, August 26-29, 1998, Abstract 194.

9. Rizzolatti G (1983). Mechanisms of selective attention in mammals In: Ewert J-P, Capranica RR \& Ingle DJ (Editors), Advances in Vertebrate Neuroethology. Plenum Press, New York, 261-297.

10. Posner $\mathrm{MI} \&$ Cohen $Y$ (1984). Components of visual orienting. In: Bouma H \& Bouwhuis DG (Editors), Attention and Performance $X$. Erlbaum Associates, Hillsdale, NJ, USA, 531-556.

11. Riggio L, Bello A \& Umiltá C (1998). Inhibitory and facilitatory effects of cue onset and offset. Psychological Research, 61: 107-
118.

12. Pratt J \& McAuliffe J (2001). The effects of onsets and offsets on visual attention. Psychological Research, 65: 185-191.

13. Judge SJ, Wurtz RH \& Richmond BJ (1980). Vision during saccadic eye movements. I. Vision interactions in striate cortex. Journal of Neurophysiology, 43: 1133-1155.

14. Reynolds JH \& Desimone R (1999). The role of neural mechanisms of attention in solving the binding problem. Neuron 24: 19 29.

15. Kastner S, De Weerd P, Desimone R \& Ungerleider LG (1998). Mechanisms of directed attention in the human extrastriate cortex as revealed by functional MRI. Science, 282: 108-111.

16. Kastner S, Pinsk MA, De Weerd P, Desimone R \& Ungerleider LG (1999). Increased activity in human visual cortex during directed attention in the absence of visual stimulation. Neuron, 22: 751761.

17. Corchs S \& Deco G (2002). Large-scale neural model for visual attention: integration of experimental single-cell and $\mathrm{fMRI}$ data Cerebral Cortex, 12: 339-348.

18. Trappenberg TP, Dorris MC \& Klein RM (2001). A model of saccade initiation based on the competitive integration of exogenous and endogenous signals in the superior colliculus. Journal of Cognitive Neuroscience, 13: 256-271.

19. Coull JT, Frith CD, Büchel C \& Nobre AC (2000). Orienting attention in time: behavioral and neuroanatomical distinction between exogenous and endogenous shifts. Neuropsychologia, 38: 808819.

20. Nobre AC (2001). Orienting attention to instants in time. Neuropsychologia, 39: 1317-1328. 